\title{
Study of Internal Motions Through N.Q.R. in 6-Chloropyridin-2-ol
}

\author{
S. P. Basavaraju, ${ }^{\mathrm{A}}$ N. Devaraj, ${ }^{\mathrm{A}}$ A. Indumathy, ${ }^{\mathrm{B}}$ K. R. Sridharan ${ }^{\mathrm{B}}$ \\ and J. Ramakrishna ${ }^{\mathrm{B}}$ \\ A Department of Physics, Bangalore University, Bangalore-560001, India. \\ ${ }^{\text {B }}$ Department of Physics, Indian Institute of Science, Bangalore-560012, India.
}

\section{Abstract}

Chlorine-35 n.q.r. has been observed for the first time in 6-chloropyridin-2-ol and its temperature dependence has been studied from $77 \mathrm{~K}$ to room temperature. The torsional frequencies and their temperature dependences have been calculated by using Bayer's theory with and without Tatsuzaki's modification.

\section{Introduction}

The nuclear quadrupole resonance (n.q.r.) of ${ }^{35} \mathrm{Cl}$ has been observed for the first time in 6-chloropyridin-2-ol and the temperature dependence of n.q.r. frequency has been investigated from $77 \mathrm{~K}$ to room temperature. A single n.q.r. line has been observed throughout.

\section{Experimental}

A home-made n.q.r. spectrometer was used to record the signals and the frequencies were measured with a BC 221 frequency meter to an accuracy of $\pm 1 \mathrm{kHz}$. The temperature variation of n.q.r. was achieved by means of a low-temperature assembly and the temperatures were measured with an iron-constantan thermocouple to an accuracy $\pm 1 \mathrm{~K}$.

\section{Methods of Calculation}

Internal motions in solids lead to averaging of the electric-field gradient (e.f.g.) and thereby render the n.q.r. frequencies temperature-dependent. Low-frequency torsional modes are the ones which contribute predominantly to the temperature dependence. $^{1,2}$

According to Bayer's theory, ${ }^{1}$ the torsional oscillations are assumed to occur about the principal axes of the e.f.g. tensor and also they are assumed to be harmonic. For very low asymmetries $(\eta)$ of the e.f.g. tensor, the motion about the e.f.g. $Z$-axis

\footnotetext{
${ }^{1}$ Bayer, H., Z. Phys., 1951, 130, 227.

${ }^{2}$ Kushida, T., J. Sci. Hiroshima Univ., Ser. A: Phys. Chem., 1955, 19, 327.
} 
has a negligible effect and hence it is ignored. The expression which relates the n.q.r. frequency and temperature for the case $I=3 / 2$ and $\eta=0$, is given by

$$
v(T)=v_{0}\left[1-\frac{3 h}{8 \pi^{2}} \sum_{i} \frac{1}{A_{i} f_{i}}\left\{\frac{1}{2}+\frac{1}{\exp \left(h f_{i} / k_{\mathrm{B}} T\right)-1}\right\}\right]
$$

( $i=X, Y, Z$, the principal axes of the e.f.g. tensor) where $v_{0}$ is the n.q.r. frequency at $0 \mathrm{~K}, A_{i}$ are the moments of inertia of the molecule about the principal axes of the e.f.g. tensor, and $f_{i}$ are the corresponding torsional frequencies.

Tatsuzaki and Yokozawa ${ }^{3}$ have shown that, for molecules for which the principal axes of the e.f.g. tensor and the principal axes of the moment of inertia are not the same, Bayer's equation has to be modified as

$$
\left.v(T)=v_{0}\left[1-\left.\frac{3 h}{8 \pi^{2}} \sum_{i} \frac{\sin ^{2} \alpha_{i}}{A_{i} f_{i}}\right|_{\frac{1}{2}}+\frac{1}{\exp \left(h f_{i} / k_{\mathrm{B}} T\right)-1}\right\}\right]
$$

( $i=1,2,3$, the principal axes of the moment of inertia) where $A_{i}$ are the moments of inertia of the molecule about its principal axes of motion, $f_{i}$ are the corresponding torsional frequencies and $\alpha_{i}$ are the angles between the principal $Z$-axis of the e.f.g. tensor and the principal axes of motion. Numerical evaluation of torsional frequencies about all the three principal axes of motion would be ideal, but such an evaluation is quite difficult. Hence, in practice, the motions of the molecule about only two principal axes that make the maximum contribution to $v(T)$ are considered, as determined by the factors $\sin ^{2} \alpha_{i} / A_{i}$.

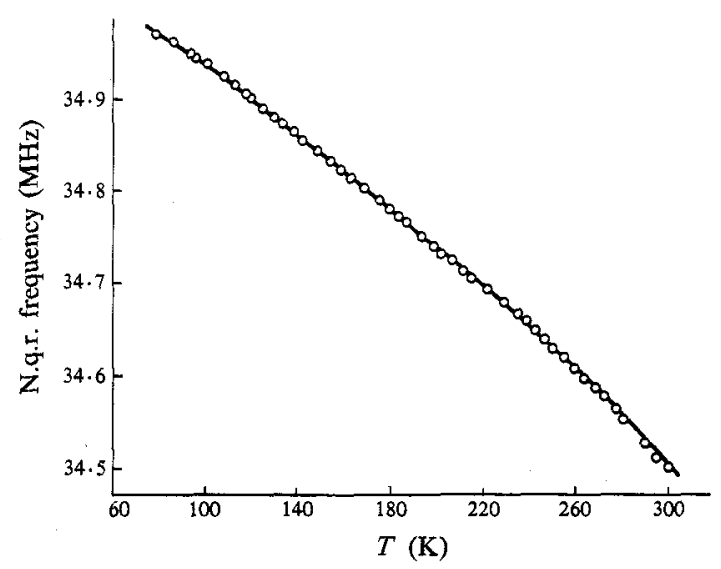

Fig. 1. Temperature dependence of n.q.r. frequency in 6-chloropyridin-2-ol.

\section{Results and Discussion}

The n.q.r. frequency of ${ }^{35} \mathrm{Cl}$ in the compound has been found to be $34.970 \mathrm{MHz}$ at $77 \mathrm{~K}$ and $34.506 \mathrm{MHz}$ at room temperature $(298 \mathrm{~K})$. The observed temperature dependence of the n.q.r. frequency is shown in Fig. 1. The resonance frequency decreases with the increase of temperature as expected and no phase transition is observed in the temperature region studied.

The parameters $A_{X}, A_{Y}, A_{1}, A_{2}, \alpha_{1}$ and $\alpha_{2}$ were calculated from the crystal structure data of Kvick and Olovsson. ${ }^{4} v_{0}$ was obtained by least-squares fitting of

${ }^{3}$ Tatsuzaki, I., and Yokozawa, Y., J. Phys. Soc. Jpn, 1957, 12, 802.

${ }^{4}$ Kvick, A., and Olovsson, I., Ark. Kemi, 1968, 30, 71. 
the experimental data of $v$ to a polynomial of order four in $T$. The values obtained for these parameters are as follows:

$\begin{array}{ccccccc}v_{0}(\mathrm{MHz}) & A_{X}\left(\mathrm{~g} \mathrm{~cm}^{2}\right) & A_{Y}\left(\mathrm{~g} \mathrm{~cm}^{2}\right) & A_{1}\left(\mathrm{~g} \mathrm{~cm}^{2}\right) & A_{2}\left(\mathrm{~g} \mathrm{~cm}^{2}\right) & \alpha_{1} & \alpha_{2} \\ 35.064 & 918 \times 10^{-40} & 630 \times 10^{-40} & 911 \times 10^{-40} & 672 \times 10^{-40} & 90^{\circ} & 71.86^{\circ}\end{array}$

The torsional frequencies were evaluated at various temperatures in the region $77-300 \mathrm{~K}$ by a numerical method ${ }^{5}$ and with a DEC- 10 computer. Fig. $2 a$ shows the variation of torsional frequency with temperature, obtained on the basis of Bayer's theory [equation (1)]. It may be seen that the variation is almost linear for the range of temperatures studied. The temperature coefficients $g_{X}$ and $g_{Y}$ of the torsional frequencies $f_{X}$ and $f_{Y}$ are calculated with the equation

$$
f_{i}=f_{i}^{\circ}\left(1-g_{i} T^{\prime}\right)
$$

with $T^{\prime}=0$ at $T=225 \mathrm{~K}$ (high-temperature approximation). $g_{X}$ and $g_{Y}$ are found to have the same value of 0.0007 .

Fig. 2. Temperature dependence of torsional frequencies in 6-chloropyridin-2-ol based on:

(a) Bayer's theory;

(b) Bayer's theory with Tatsuzaki's correction.

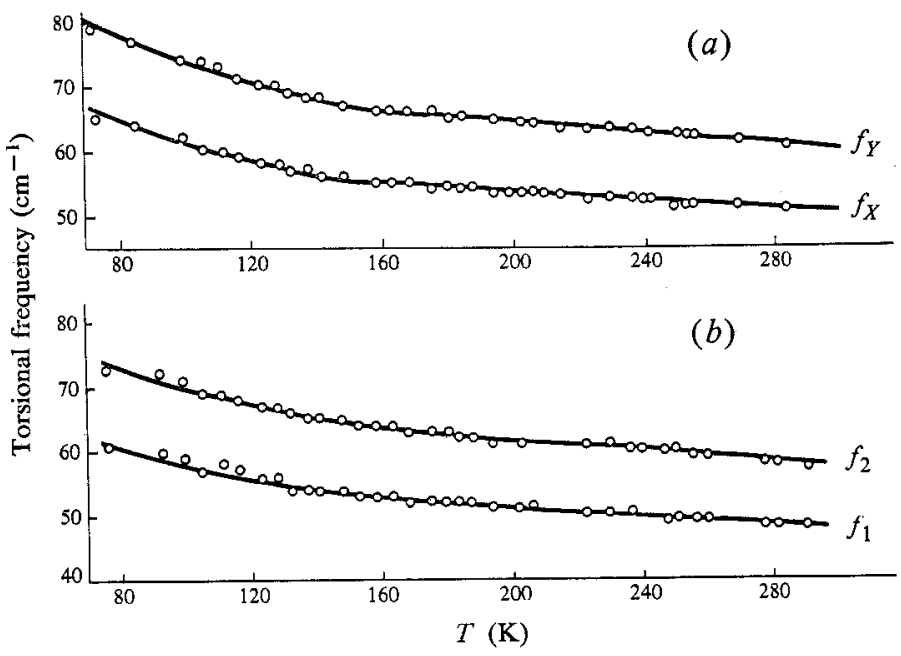

The temperature variation of the torsional frequency obtained on the basis of Bayer's theory incorporating Tatsuzaki's correction [equation (2)] is shown in Fig. $2 b$. The values of the temperature coefficients $g_{1}$ and $g_{2}$ of the torsional frequencies $f_{1}$ and $f_{2}$ at $225 \mathrm{~K}$ and their weighted averages $g$ were obtained as $0.0007,0.0009$ and 0.0008 respectively.

The experimental results were also analysed by means of Brown's method ${ }^{6,7}$ by fitting the experimental data in the high-temperature region to a parabola centred at $T_{0}=225 \mathrm{~K}$, and a value of 0.0005 was obtained for $g$. This value compares well with those obtained by the numerical method.

The values obtained for torsional frequencies by Bayer's method lie in the range $50-80 \mathrm{~cm}^{-1}$, and those obtained by employing Tatsuzaki's correction lie in the range $45-75 \mathrm{~cm}^{-1}$; this shows that the effect of Tatsuzaki's correction is rather small in

5 Vijaya, M. S., and Ramakrishna, J., Mol. Phys., 1970, 19, 131.

${ }^{6}$ Brown, R. J. C., J. Chem. Phys., 1960, 32, 116.

7 Chandramani, R., Devaraj, N., Sastry, V.S. S., and Ramakrishna, J., Aust. J. Chem., 1976, $29,2363$. 
this case. The torsional frequencies obtained are in the expected range of lowfrequency modes in molecular crystals, viz. $20-150 \mathrm{~cm}^{-1}$ (see for example Stidham ${ }^{8}$ ). It would be interesting to compare these values of frequencies with Raman and infrared data when they become available.

\section{Acknowledgment}

One of the authors (N.D.) is grateful to the University Grants Commission, Government of India, for the award of a research grant.

Manuscript received 26 June 1981

${ }^{8}$ Stidham, H. D., J. Chem. Phys., 1968, 49, 2041. 